\title{
Patient Perceptions of Wearable Face-Mounted Computing Technol- ogy and the Effect on the Doctor- Patient Relationship
}

Micah T. Prochaska'; Valerie G. Press'; David O. Meltzer'; Vineet M. Arora

'Department of Medicine, The University of Chicago, Chicago, IL.

\section{Keywords}

Technology, mobile health, face-mounted technology, Google Glass ${ }^{\mathrm{TM}}$, doctor-patient relationship

\section{Summary}

Background: Wearable face-mounted computers such as Google Glass ${ }^{\top M}$, Microsoft HoloLens ${ }^{\top M}$, and Oculus' Rift ${ }^{\circledR}$, are increasingly being tested in hospital care. These devices challenge social etiquette, raise privacy issues, and may disrupt the intimacy of the doctor patient relationship. We aimed to determine patients' perception of and their privacy concerns with an archetype of wearable face-mounted computer devices, Google Glass.

Methods: Hospitalized inpatients were asked about their familiarity with Glass, how comfortable they would be and if they would be concerned about privacy if their physician wore Glass, if the use of Glass would affect their trust in their physician, and if they would want their physician to wear Glass if it improved their care.

Results: Most (73\%) respondents were unfamiliar with Glass, though $64 \%$ would be comfortable if their doctor wore Glass. Under half ( $46 \%)$ of respondents were concerned about privacy with the use of Glass. Seventy-six percent (76\%) of respondents stated their doctor wearing Glass would not affect their trust in their doctor. Patients concerned about their privacy were less likely to trust their doctor if their doctor wore Glass ( $17 \%$ vs. $0 \%, p<0.01)$. Sixty-five percent $(65 \%)$ of respondents would want their doctor to wear Glass if it improved their care.

Conclusion: Most patients appear open to and would want their doctor to use face-mounted wearable computers such as Glass, even when unfamiliar with this technology. While some patients expressed concerns about privacy, patients were much less concerned about wearable technologies affecting the trust they have in their physician.

\section{Correspondence to:}

Micah T. Prochaska, MD, MS

University of Chicago

5841 S. Maryland Avenue, MC 5000

Chicago, IL 60637

Email: mprochas@medicine.bsd.uchicago.edu

Phone: $773-702-6988$

Fax: 773-795-7398
Appl Clin Inform 2016; 7: 946-953

http://dx.doi.org/10.4338/ACl-2016-06-LE-0094

received: June 14, 2016

accepted: August 26, 2016

published: October 12, 2016

Citation: Prochaska MT, Press VG, Meltzer DO, Arora

VM. Patient perceptions of wearable face-mounted

computing technology and the effect on the doctor-patient relationship. Appl Clin Inform 2016; 7: 946-953

http://dx.doi.org/10.4338/ACI-2016-06-LE-0094

\section{Funding}

Dr. Prochaska received funding the Agency for Healthcare Research and Quality (K12 Patient Centered Outcome Research Award).

Dr. Press received funding from the National Heart Lung and Blood Institute (NIH K23HL1181510).

Dr. Meltzer received funding from the National Institute of Aging (K24 AG031326-01) and from the National Center for Advancing Translational Science (2UL1TR000430-06). 


\section{Dear Editor,}

There has been increased interest in the development and use of face-mounted wearable computers in health care. Examples of such technologies include Google Glass (Glass) ${ }^{\mathrm{TM}}$, the newer Microsoft HoLolens $^{\mathrm{TM}}$, and Oculus' Rift ${ }^{\circledR}[1-3]$. Entrepreneurs believe these devices can add value to and even improve health care delivery [4-7], and a previously released version of Glass has already been used to monitor vital signs, search health records, offer video streaming of operating rooms, and provide medical education opportunities [8-13]. However, because these devices are conspicuous, they challenge established social etiquette [14], and they are therefore distinct from technology like tablets, cellphones, or other wearable technologies that have been integrated into everyday clinical practice. While face-mounted computers are being touted for use in health care, it is important to know how patients perceive these devices, and if they disrupt the doctor-patient relationship. For example, patients may have reservations about how face-mounted computers protect the privacy of their sensitive health information, since these devices on-demand video with upload to cloud capabilities [10].

Therefore, patients' acceptance of these devices is a critical barrier to their widespread implementation and adoption into patient care, and it is important to understand if patients would feel comfortable with physicians wearing these devices and if they have concerns that these devices may undermine their privacy. We aimed to determine patients' perception of and their privacy concerns with an archetype of a wearable face-mounted computer, Glass.

\section{Methods}

All hospitalized general medicine inpatients were approached for written consent for the University of Chicago Hospitalist Project, an established research infrastructure for studying hospitalized patients at the University of Chicago [15]. Among patients consenting to participate in the Hospitalist Project, patients were eligible and asked to participate in this project if they were 18 years or older and English speaking. A research assistant read consenting patients a description of Glass, including that "Glass is a wearable computer with an optical head-mounted display that is developed by Google", and that "Glass can take videos and pictures, make telephone calls, and play music." Patients were then shown a picture with a physician wearing the Glass face-mounted computer spectacles ( Figure 1) and were asked:

1. How familiar are you with Google Glass?

2. How comfortable would you be if one of your doctors were wearing Google Glass?

3. Google Glass has the ability to take photos and record video, if your doctor was wearing Google Glass how concerned would you be about your privacy?

4. How would Google Glass affect your trust in your doctor?

5. If your doctor said Google Glass helped them to deliver better care would you want them to wear Google Glass?

Responses for the first 3 questions used a Likert scale; question 4 responses included: "more likely to trust your doctor," "less likely to trust your doctor," "no change" or "I don't know;" question 5 responses included: “yes," "no," or "I don’t know" (> Figure 2). Results for each question were dichotomized in order to test for categorical associations between questions using Fishers exact test. The University of Chicago institutional review board reviewed and approved this study.

\section{Results}

During the study period from 5/7/2014 through 1/31/2015, 92 patients were eligible and approached for participation. Eighty-six [93\% (86/92)] patients consented and completed the study with 6 [7\% (6/92)] patients refusing to participate. The mean age (s.d.) was 46 (14.7) years old with a range from age 19 to age 88. Fifty-three percent [53\% (46/86)] of participants were female. Self-reported race was 69\% (59/86) African-American, 21\% (18/86) Caucasian, 4\% (4/86) “other", and 6\% (5/86) “don't know or refused". Seven percent $[7 \%(6 / 86)]$ of participants had less than a twelfth grade education, 
$35 \%(30 / 86)$ completed twelfth grade, 35\% (30/86) had some college education, and 18\% (16/86) had a college education or more $(-$ Table 1$)$.

Most participants [73\% (63/86)] were unfamiliar with Glass, yet two-thirds [64\% (55/86)] stated they would be comfortable if their doctor wore Glass. The video and photo capability of Glass divided participants with respect to concern for their privacy. Forty-six percent [46\% (39/85)] would be concerned, $47 \%$ (40/85) would not be concerned, and 7\% (6/85) would be neither concerned nor unconcerned about their privacy if their doctor were to wear Glass (one participant refused question). Three-quarters [76\% (65/86)] of participants felt that their doctor wearing Glass would not change their trust in their doctor, with only $9 \%$ (8/86) reporting they would trust their doctor less. Among participants who had privacy concerns about Glass, $18 \%$ were less likely to trust their doctor wearing Glass, while among participants who had no privacy concerns about Glass $0 \%$ reported trusting their doctor less $(\mathrm{p}<0.01)$ ( $>$ Figure 3$)$. There were no associations between patient age or race, and familiarity with Glass, or privacy or trust in their doctor were their doctor to wear glass. Two-thirds [65\% (56/86) of participants would want their doctor to wear Glass if it improved their care.

\section{Discussion}

Among the hospitalized patients we studied, most were open to their doctor using a wearable facemounted computer, even when unfamiliar with this technology. Although many patients had concerns about privacy, very few expressed concern that it would affect their trust in their doctor. This suggests that patients' trust in their physician may mediate the privacy concerns patients have with face-mounted technology. Further, neither privacy concerns nor concerns with the social appropriateness of face-mounted computers appear to hinder patients trust in their physician, which is integral to maintaining the doctor-patient relationship. Additionally, despite patients' privacy concerns, the majority of patients would want their doctor to use such technology if it improved their care. Therefore, physician trust and the potential for improved care may trump privacy concerns that patients have when it comes to the use of face-mounted computers for clinical care.

Our study is limited as a single urban academic medical center, and it is possible that our results may not be generalizable to other institutions. Additionally, Google Glass was the only facemounted computer available at the time of the study design, and it is possible that patients' perception of other now available face-mounted computers may be different than Google Glass, or that patients' initial perceptions may lead to premature opinions.

We believe we are the first study to directly ask patients how they perceive face-mounted computers and their use in a patient care setting. Our findings are important because as the technology grows and newer products become available (such as Microsoft HoLolens, Oculus Rift, and Google Glass Version 2) there are a wide variety of potential uses for face mounted computers across clinical and health care settings. While patients' perceptions of these devices may vary in different clinical settings (inpatient encounter, outpatient encounter, operating room or procedural suite), the issues of physician trust and the privacy of protected health information are universal and important considerations for patients with any new technology whose uses are adapted into health care. To accept face-mounted computers as a routine part of clinical care patients will need to trust how clinicians use these devices, be assured that their private health information is protected, and assent to any initial social awkward interactions and feelings these devices may engender. Therefore, our results are a first step towards understanding how patients may initially perceive the adaption of face-mounted computers, which are a new and relatively unknown form of technology, into use for clinical care. Future studies should focus on how face-mounted computers are perceived by a broader range of patients in different clinical care settings, and they should address specifically patients' perception of and the actual security concerns of these devices when collecting protected patient health information. Additionally, future studies should explore if newer versions of face-mounted computers can actually improve the efficiency and/or quality of care delivered to patients. 


\section{Clinical Relevance}

While developers and entrepreneurs are excited by how face-mounted computing technology can to add value to health care, it is unknown how patients may perceive such devices when used by their physician. To be accepted as a new technology in health care, it is important to understand how patients perceive face-mounted computers to affect their privacy or disrupt the intimacy of the doctor patient relationship. This project is instructive because face-mounted computing is a rapidly growing technology with health care as an intended industry for use and application. Since face mounted computers are so conspicuous, the adaption of these devices poses challenges distinct from other technologies that have been adapted into clinical practice (tablets, cellphones)

\section{Conflict of Interest}

None of the authors have any conflicts of interests, including financial interests, activities, relationships or affiliations.

\section{Human Subjects}

The procedures used in this study were reviewed and approved by the University of Chicago institutional review board. This study is in compliance with the ethics standards of the responsible committee on human experimentation and with the World Medical Association Declaration of Helsinki on Ethical Principles for Medical Research Involving Human Subjects.

\section{Acknowledgements}

We would like to thank John Kim, Eddie Kim, Nicole Twu, and the staff of the Hospitalist Project for their assistance with this project. 
Fig. 1 Google Glass Photo

Google Glass is a wearable computer with an optical head-mounted display that is developed by Google. Currently Google Glass remains under development with certain individuals being granted the opportunity test and develop new software to use with Google Glass. Among other things, "Glass" can take videos and pictures, make telephone calls, and play music. In medicine, some physicians and health systems are interested in using "Glass" to improve the efficiency and quality of care, as well as using "Glass" to ultimately improve the patients' experience. (RA holds picture of Doctor wearing Google Glass)

1. How familiar are you with Google Glass?
a. Very Familiar
b. Somewhat Familiar
c. Neither Familiar or Unfamiliar
d. Somewhat Unfamiliar
e. Very Unfamiliar

2. How comfortable would you feel if one of your doctors was wearing Google Glass?
a. Very Comfortable
b. Somewhat Comfortable
c. Neither Comfortable or Uncomfortable
d. Somewhat Uncomfortable

e. Very Uncomfortable

3. Google Glass has the ability to take photos and record video. If your doctor was wearing Google Glass, how concerned about your privacy would you be?
a. Very Concerned
b. Somewhat Concerned
c. Neither Concerned nor Unconcerned
d. Somewhat Unconcerned

e. Very Unconcerned

4. How would Google Glass affect your trust in your doctor?
a. More likely to trust your docto
b. No change
c. Less likely to trust your doctor
d. I don't know

5. If your doctor said Google Glass helped them deliver better care, would you want them to wear Google Glass?
a. Yes
b. No
c. I don't know

Fig. 2 Face-mounted computer survey administered to patients 


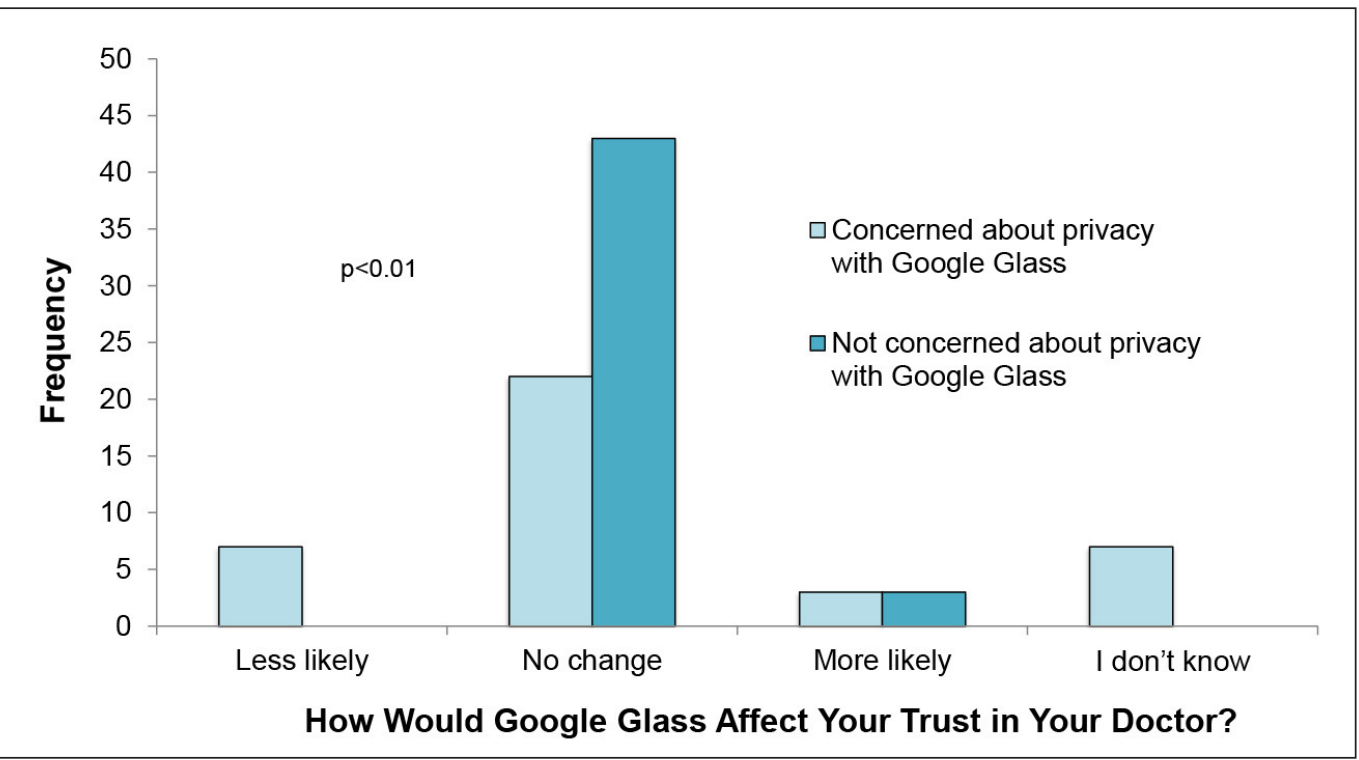

Fig. 3 Concern for Privacy vs. Trust in Physician 


\begin{tabular}{|c|c|}
\hline Total N=86 & N (\%) \\
\hline Female & $46(53)$ \\
\hline Age-Mean \pm SD (years) & $46 \pm 14.7$ \\
\hline \multicolumn{2}{|l|}{ Race } \\
\hline $\begin{array}{l}\text { Black or African American } \\
\text { White } \\
\text { Other } \\
\text { Don't know/refused }\end{array}$ & $\begin{array}{l}59(69) \\
18(21) \\
4(4) \\
5(6)\end{array}$ \\
\hline \multicolumn{2}{|l|}{ Education Level } \\
\hline $\begin{array}{l}<12 \text { Grade } \\
\text { Completed grade } 12 \\
\text { Some College } \\
\text { College and/or Post Graduate } \\
\text { Don't know/refused }\end{array}$ & $\begin{array}{l}6(7) \\
30(35) \\
30(35) \\
16(18) \\
4(5)\end{array}$ \\
\hline
\end{tabular}

Table 1 Patient Characteristics 


\section{References}

1. Case Western Reserve, Cleveland Clinic Collaborate with Microsoft on Earth-Shattering Mixed-Reality Technology for Education. [cited 2015 Aug 27]. Available from: http://www.case.edu/hololens/

2. Developers will get their hands on Microsoft's HoloLens within a year. PCWorld. 2015 [cited 2015 Aug 5]. Available from: http://www.pcworld.com/article/2955665/windows/developers-will-get-their-hands-onmicrosofts-hololens-within-a-year.html

3. Barr A. Google Quietly Distributes New Version of Glass Aimed at Workplaces. Wall Street Journal. 2015 Jul 31 [cited 2015 Aug 5]; Available from: http://www.wsj.com/articles/google-quietly-distributes-newversion-of-glass-aimed-at-workplaces-1438283319

4. Technology in Academic Medicine: Medicine Takes a Closer Look at Google Glass - April 2014 - AAMC Reporter - Newsroom - AAMC. [cited 2015 Jan 13]. Available from: https://www.aamc.org/newsroom/re porter/april2014/378176/technology.html

5. How This Google Glass Startup Is Saving Doctors Time. Entrepreneur. [cited 2015 Jan 13]. Available from: http://www.entrepreneur.com/article/239183

6. Stewart J, Billinghurst M. A wearable navigation display can improve attentiveness to the surgical field. Int J Comput Assist Radiol Surg 2016 ; 11(6): 1193-1200.

7. Gupta S, Boehme J, Manser K, Dewar J, Miller A, Siddiqui G, Schwaitzberg SD. Does Wearable Medical Technology With Video Recording Capability Add Value to On-Call Surgical Evaluations? Surg Innov 2016 Jun 22;

8. Emergency providers see big potential for Google Glass. ED Manag Mon Update Emerg Dep Manag 2014; 26(5): 55-58.

9. Tully J, Dameff C, Kaib S, Moffitt M. Recording Medical Students' Encounters With Standardized Patients Using Google Glass: Providing End-of-Life Clinical Education. Acad Med J Assoc Am Med Coll 2014 Dec 30 ;

10. Muensterer OJ, Lacher M, Zoeller C, Bronstein M, Kübler J. Google Glass in pediatric surgery: An exploratory study. Int J Surg 2014; 12(4): 281-289.

11.Zhang Z, Pei J, Wang D, Gan Q, Ye J, Yue J, Wang B, Povoski SP, Martin EW Jr, Hitchcock CL, Yilmza A, Tweedle MF, Shao P, Xu RX. A Wearable Goggle Navigation System for Dual-Mode Optical and Ultrasound Localization of Suspicious Lesions: Validation Studies Using Tissue-Simulating Phantoms and an Ex Vivo Human Breast Tissue Model. PloS One 2016; 11(7): e0157854.

12.Zahl DA, Schrader SM, Edwards PC. Student perspectives on using egocentric video recorded by smart glasses to assess communicative and clinical skills with standardised patients. Eur J Dent Educ Off J Assoc Dent Educ Eur 2016 Jul 6;

13. Vaughn J, Lister M, Shaw RJ. Piloting Augmented Reality Technology to Enhance Realism in Clinical Simulation. Comput Inform Nurs CIN 2016 Jun 2;

14. Gross A. What's the Problem with Google Glass? The New Yorker 2014 [cited 2015 Jan 13]. Available from: http://www.newyorker.com/business/currency/whats-the-problem-with-google-glass

15. Meltzer D, Manning WG, Morrison J, Shah MN, Jin L, Guth T, Levinson W. Effects of Physician Experience on Costs and Outcomes on an Academic General Medicine Service: Results of a Trial of Hospitalists. Ann Intern Med 2002; 137(11): 866-874. 